\title{
Rapidity dependence of transverse-momentum multiplicity correlations
}

\author{
Adam Bzdak* \\ AGH University of Science and Technology, \\ Faculty of Physics and Applied Computer Science, \\ 30-059 Kraków, Poland
}

\begin{abstract}
Following previous work [1], we propose to analyze the rapidity dependence of transverse momentum and transverse-momentum multiplicity correlations. We demonstrate that the orthogonal polynomial expansion of the latter has the potential to discriminate between models of particle production.
\end{abstract}

\section{INTRODUCTION}

One of the central problems in high-energy hadronic collisions is to understand the longitudinal structure of systems created in proton-proton $(\mathrm{p}+\mathrm{p})$, proton-nucleus $(\mathrm{p}+\mathrm{A})$ and nucleus-nucleus $(\mathrm{A}+\mathrm{A})$ collisions.

Not long ago, it was argued that an event-by-event long-range fluctuation of the fireball rapidity distribution results in rather peculiar two- and multi-particle rapidity correlations [1, 2]. Recent measurement by the ATLAS Collaboration at the $\mathrm{LHC}[3]$ revealed new and rather unexpected scaling results on asymmetric rapidity fluctuations in $\mathrm{p}+\mathrm{p}, \mathrm{p}+\mathrm{A}$ and $\mathrm{A}+\mathrm{A}$ interactions. Recently, this problem has drawn a noticeable theoretical [4-11] and experimental [3, 12, 13] interest, see also [14 23] for recent related studies.

To summarize the main idea, the single-particle rapidity distribution in each event, $N(y)$, can be written as

$$
\frac{N(y)}{\langle N(y)\rangle}=1+a_{0}+a_{1} y+\ldots
$$

where $a_{0}$ describes the rapidity independent fluctuation of the fireball. $a_{1}$ represents the fluctuating long-range forward-backward rapidity asymmetry 1 This coefficient can be driven for example by the difference in the number of left- and right-going sources of particles, e.g., wounded nucleons [24, 25]. $\langle N(y)\rangle$ is the average rapidity distribution in a given centrality class. By definition $\left\langle a_{i}\right\rangle=0$.

It is straightforward to calculate the two-particle rapidity correlation [1]

$$
\frac{C\left(y_{1}, y_{2}\right)}{\left\langle N\left(y_{1}\right)\right\rangle\left\langle N\left(y_{2}\right)\right\rangle}=\left\langle a_{0}^{2}\right\rangle+\left\langle a_{1}^{2}\right\rangle y_{1} y_{2}+\ldots
$$

where 2

$$
C\left(y_{1}, y_{2}\right)=\left\langle N\left(y_{1}\right) N\left(y_{2}\right)\right\rangle-\left\langle N\left(y_{1}\right)\right\rangle\left\langle N\left(y_{2}\right)\right\rangle .
$$

\footnotetext{
* E-Mail:bzdak@fis.agh.edu.pl

${ }^{1}$ We are not interested in statistical fluctuations, which can also generate nonzero values of $a_{i}$. These are removed by measuring correlation functions.

${ }^{2}$ For clarity we skip $\left\langle a_{0} a_{1}\right\rangle$, which vanishes in symmetric (e.g. $\mathrm{p}+\mathrm{p})$ collisions.
}

As seen in Eq. (2), the long-range fluctuation of the fireball rapidity distribution, parameterized by fluctuating $a_{i}$, results in rather nontrivial correlations. The first term corresponds to a well-known rapidity independent multiplicity fluctuation, and it can be driven by, e.g., the impact parameter or volume fluctuation. The second term, $\sim y_{1} y_{2}$, is related to the fluctuating forward-backward asymmetry in rapidity. In the wounded nucleon model [24, 25] $\left\langle a_{1}^{2}\right\rangle \sim\left\langle\left(w_{L}-w_{R}\right)^{2}\right\rangle$, where $w_{L(R)}$ is the number of left(right)-going wounded nucleons [1]. Recently, the ATLAS Collaboration observed $\left\langle a_{1}^{2}\right\rangle y_{1} y_{2}$ in the twoparticle rapidity correlation functions measured in $\mathrm{p}+\mathrm{p}$, $\mathrm{p}+\mathrm{Pb}$ and $\mathrm{Pb}+\mathrm{Pb}$ collisions [3]. They found that at a given event multiplicity $N_{\mathrm{ch}},\left\langle a_{1}^{2}\right\rangle$ approximately scales with $1 / N_{\mathrm{ch}}$ and numerically is very similar for all colliding systems $3^{3}$ This surprising result still calls for a quantitative explanation.

\section{TRANSVERSE-MOMENTUM MULTIPLICITY CORRELATIONS}

It is proposed here to analyze, in a similar way, the rapidity dependence of transverse momentum and especially transverse-momentum multiplicity correlations.

Analogously to Eq. (1) we have

$$
\frac{P_{t}(y)}{\left\langle P_{t}(y)\right\rangle}=1+b_{0}+b_{1} y+\ldots
$$

where $P_{t}(y)$ is the average (in one event) transverse momentum of particles in a given rapidity bin $y$

$$
P_{t}(y)=\frac{1}{N} \sum_{i=1}^{N} p_{t}^{(i)}
$$

where $N$ is the number of particles (in a given event) at $y$. Here $p_{t}^{(i)}$ is the transverse momentum magnitude of the $i$-th particle. $\left\langle P_{t}(y)\right\rangle$ is the average of $P_{t}(y)$ over

\footnotetext{
3 One would expect rather different results in, e.g., peripheral $\mathrm{Pb}+\mathrm{Pb}$ and ultracentral $\mathrm{p}+\mathrm{p}$ collisions $\left(N_{\mathrm{ch}} \sim 150[3]\right)$. The ATLAS result suggests that the number of particle sources (at a given $N_{\mathrm{ch}}$ ) and their fluctuations are actually similar in all measured systems.
} 
many events in a given centrality class. We note that $\left\langle b_{i}\right\rangle=0$, in close analogy to the $a_{i}$ coefficients.

The transverse momentum correlation function (studied extensively in the literature for rather different reasons, see, e.g., [26-28]) reads

$$
\frac{C_{[P, P]}\left(y_{1}, y_{2}\right)}{\left\langle P_{t}\left(y_{1}\right)\right\rangle\left\langle P_{t}\left(y_{2}\right)\right\rangle}=\left\langle b_{0}^{2}\right\rangle+\left\langle b_{1}^{2}\right\rangle y_{1} y_{2}+\ldots
$$

where

$$
C_{[P, P]}\left(y_{1}, y_{2}\right) \equiv\left\langle P_{t}\left(y_{1}\right) P_{t}\left(y_{2}\right)\right\rangle-\left\langle P_{t}\left(y_{1}\right)\right\rangle\left\langle P_{t}\left(y_{2}\right)\right\rangle .
$$

The first term in Eq. (6) describes an event-by-event rapidity independent transverse momentum fluctuation. This could be driven for example by an event-by-event long-range multiplicity fluctuation (if event multiplicity is correlated with $P_{t}$ ). The second term describes the forward-backward rapidity asymmetric transverse momentum fluctuation. A possible source of this effect is the forward-backward fireball multiplicity fluctuation.

It would be especially interesting to measure an eventby-event relation between $a_{i}$ and $b_{i}$ coefficients. In order to do this, one can construct a simple correlation function

$$
C_{[N, P]}\left(y_{1}, y_{2}\right) \equiv\left\langle N\left(y_{1}\right) P_{t}\left(y_{2}\right)\right\rangle-\left\langle N\left(y_{1}\right)\right\rangle\left\langle P_{t}\left(y_{2}\right)\right\rangle,
$$

witch correlates multiplicity and transverse momentum, see, e.g., [28]. This results in

$$
\frac{C_{[N, P]}\left(y_{1}, y_{2}\right)}{\left\langle N\left(y_{1}\right)\right\rangle\left\langle P_{t}\left(y_{2}\right)\right\rangle}=\left\langle a_{0} b_{0}\right\rangle+\left\langle a_{1} b_{1}\right\rangle y_{1} y_{2}+\ldots
$$

The meaning of mixed coefficients $\left\langle a_{i} b_{k}\right\rangle$ is easy to understand. The first term describes the relation between rapidity independent fluctuation of multiplicity and transverse momentum. The second term is particularly interesting and describes how rapidity asymmetry in multiplicity is related to rapidity asymmetry of transverse momentum. If the particle multiplicity and $P_{t}$ are not correlated then $\left\langle a_{i} b_{k}\right\rangle=\left\langle a_{i}\right\rangle\left\langle b_{k}\right\rangle=0$.

In general, the above correlation functions can be expanded in terms of the orthogonal polynomials [1]. For example

$$
\frac{C_{[N, P]}\left(y_{1}, y_{2}\right)}{\left\langle N\left(y_{1}\right)\right\rangle\left\langle P_{t}\left(y_{2}\right)\right\rangle}=\sum_{i, k}\left\langle a_{i} b_{i}\right\rangle T_{i}\left(y_{1}\right) T_{k}\left(y_{2}\right),
$$

with $T_{i}$ being, e.g., the Chebyshev or the Legendre polynomials [1, 4], and analogously for Eqs. (21) and (6).

\section{DISCUSSION AND CONCLUSIONS}

Several comments are in order.

Consider a set of events with $a_{1}>0$, i.e., the fireball multiplicity is larger for positive $y, N(y) \sim a_{1} y$. The question is what is the rapidity dependence of the transverse momentum in this case. If $P_{t}$ is also larger for positive $y$ then $b_{1}>0$ and thus $\left\langle a_{1} b_{1}\right\rangle>0$. This scenario is expected in a typical hydrodynamical framework, see, e.g., [29].

For example, in the color glass condensate (CGC) framework [30, 31] one could expect a rather different conclusion. Consider a proton-proton event, where the two protons are characterized by different saturation scales, $Q_{1}$ and $Q_{2}$. The importance of such fluctuations was recently discussed in Refs. [9, 32 35]. Here $Q_{1}^{2}=Q_{0,1}^{2} e^{+\lambda y}$ and $Q_{2}^{2}=Q_{0,2}^{2} e^{-\lambda y}$ with $\lambda \sim 0.3$, see, e.g., 36]. We choose $Q_{0,1}>Q_{0,2}$ so that in a given rapidity bin, say $|y|<2, Q_{1}>Q_{2}$, resulting in rapidity asymmetric $N(y)$. In this case [29, 37]

$$
\begin{aligned}
& N(y) \sim S_{t} Q_{2}^{2}\left[2+\ln \left(Q_{1}^{2} / Q_{2}^{2}\right)\right], \\
& P_{t}(y) \sim \frac{2 Q_{1}-\frac{2}{3} Q_{2}}{1+\ln \left(Q_{1} / Q_{2}\right)},
\end{aligned}
$$

that is, in CGC the multiplicity is driven by the smaller scale in contrast to the transverse momentum controlled by the larger one [37]. Since $Q_{1}^{2} \sim e^{+\lambda y}$ and $Q_{2}^{2} \sim e^{-\lambda y}$, the multiplicity and the transverse momentum rapidity asymmetries have different signs. If $N(y)$ is growing with rapidity, then $P_{t}(y)$ is decreasing with $y$. Consequently $a_{1} \gtrless 0$ means $b_{1} \lessgtr 0$ and $\left\langle a_{1} b_{1}\right\rangle<0$. Clearly, this observation should be treated with caution and more detailed calculations are warranted, see, e.g., 38, 39]. The sole purpose of this exercise was to demonstrate that the sign of $\left\langle a_{1} b_{1}\right\rangle$ is not at all obvious, and could potentially discriminate between different models of particle production.

As discussed earlier, the ATLAS Collaboration reported a surprising scaling of $\left\langle a_{1}^{2}\right\rangle$ in $\mathrm{p}+\mathrm{p}, \mathrm{p}+\mathrm{Pb}$ and $\mathrm{Pb}+\mathrm{Pb}$ collisions [3]. At a given event multiplicity $N_{\mathrm{ch}}$, $\left\langle a_{1}^{2}\right\rangle$ scales with $1 / N_{\mathrm{ch}}$ and is quantitatively very similar for all three systems. It would be very interesting to see if $\left\langle b_{1}^{2}\right\rangle$ and $\left\langle a_{1} b_{1}\right\rangle$ satisfy similar scaling.

Obviously, it would be also desired to study higher order correlation functions [2, 40].

An alternative way to analyze the above correlation functions is the principal component analysis, discussed in Ref. [41].

In conclusion, it is proposed to analyze the rapidity dependence of transverse momentum and in particular transverse-momentum multiplicity correlation functions using the orthogonal polynomial expansion. A careful study of the coefficients $\left\langle a_{i}^{2}\right\rangle,\left\langle b_{i}^{2}\right\rangle$ and $\left\langle a_{i} b_{k}\right\rangle$ could potentially discriminate between different models of particle production, and reveal detailed information on the longitudinal structure of systems created in $\mathrm{p}+\mathrm{p}, \mathrm{p}+\mathrm{A}$ and $\mathrm{A}+\mathrm{A}$ collisions.

\section{Acknowledgments}

We thank Piotr Bożek and Volker Koch for useful comments. This work is supported by the Ministry of Science and Higher Education (MNiSW) and by the National Science Centre, Grant No. DEC-2014/15/B/ST2/00175, and in part by DEC-2013/09/B/ST2/00497. 
[1] A. Bzdak and D. Teaney, Phys. Rev. C 87, no. 2, 024906 (2013) arXiv:1210.1965 [nucl-th]].

[2] A. Bzdak and P. Bozek, Phys. Rev. C 93, no. 2, 024903 (2016) arXiv:1509.02967 [hep-ph]].

[3] M. Aaboud et al. [ATLAS Collaboration], Phys. Rev. C 95, no. 6, 064914 (2017) arXiv:1606.08170 [hep-ex]].

[4] J. Jia, S. Radhakrishnan and M. Zhou, Phys. Rev. C 93, no. 4, 044905 (2016) arXiv:1506.03496 [nucl-th]].

[5] P. Bozek, W. Broniowski and A. Olszewski, Phys. Rev. C 92, no. 5, 054913 (2015) arXiv:1509.04124 [nucl-th]].

[6] A. Monnai and B. Schenke, Phys. Lett. B 752, 317 (2016) arXiv:1509.04103 [nucl-th]].

[7] W. Broniowski and P. Bozek, Phys. Rev. C 93, no. 6, 064910 (2016) arXiv:1512.01945 [nucl-th]].

[8] B. Schenke and S. Schlichting, Phys. Rev. C 94, no. 4, 044907 (2016) arXiv:1605.07158 [hep-ph]].

[9] A. Bzdak and K. Dusling, Phys. Rev. C 93, no. 3, 031901 (2016) arXiv:1511.03620 [hep-ph]]; Phys. Rev. C 94, 044918 (2016) arXiv:1607.03219 [hep-ph]].

[10] W. Ke, J. S. Moreland, J. E. Bernhard and S. A. Bass, arXiv:1610.08490 [nucl-th].

[11] R. He, J. Qian and L. Huo, arXiv:1702.03137 [nucl-th].

[12] S. Jowzaee [STAR Collaboration], talk at the the XXVI international conference on ultrarelativistic heavy-ion collisions (Quark Matter 2017), Chicago, 2017.

[13] Saehanseul Oh [ALICE Collaboration], poster at the the XXVI international conference on ultrarelativistic heavyion collisions (Quark Matter 2017), Chicago, 2017.

[14] P. Bozek, W. Broniowski and J. Moreira, Phys. Rev. C 83, 034911 (2011) arXiv:1011.3354 [nucl-th]].

[15] A. Bialas and K. Zalewski, Nucl. Phys. A 860, 56 (2011) arXiv:1101.1907 [hep-ph]].

[16] A. Bialas, A. Bzdak and K. Zalewski, Phys. Lett. B 710, 332 (2012) arXiv:1107.1215 [hep-ph]].

[17] L. P. Csernai, G. Eyyubova and V. K. Magas, Phys. Rev. C 86, 024912 (2012) Erratum: [Phys. Rev. C 88, 019902 (2013)] arXiv:1204.5885 [hep-ph]].

[18] L. Pang, Q. Wang and X. N. Wang, Phys. Rev. C 86, 024911 (2012) arXiv:1205.5019 [nucl-th]].

[19] V. Vovchenko, D. Anchishkin and L. P. Csernai, Phys. Rev. C 88, no. 1, 014901 (2013) arXiv:1306.5208 [nuclth]].

[20] A. Olszewski and W. Broniowski, Phys. Rev. C 92, no. 2, 024913 (2015) arXiv:1502.05215 [nucl-th]].

[21] J. Casalderrey-Solana, M. P. Heller, D. Mateos and W. van der Schee, Phys. Rev. Lett. 112, no. 22, 221602
(2014) arXiv:1312.2956 [hep-th]].

[22] V. Vechernin, Nucl. Phys. A 939, 21 (2015).

[23] L. G. Pang, H. Petersen, G. Y. Qin, V. Roy and X. N. Wang, Eur. Phys. J. A 52, no. 4, 97 (2016) arXiv:1511.04131 [nucl-th]].

[24] A. Bialas, M. Bleszynski and W. Czyz, Nucl. Phys. B 111, 461 (1976).

[25] A. Bialas and W. Czyz, Acta Phys. Polon. B 36, 905 (2005) hep-ph/0410265.

[26] S. Gavin and M. Abdel-Aziz, Phys. Rev. Lett. 97, 162302 (2006) nucl-th/0606061.

[27] S. Gavin, G. Moschelli and C. Zin, Phys. Rev. C 94, no. 2, 024921 (2016) arXiv:1606.02692 [nucl-th]].

[28] M. A. Braun, R. S. Kolevatov, C. Pajares and V. V. Vechernin, Eur. Phys. J. C 32, 535 (2004) hep-ph/0307056.

[29] P. Bozek, A. Bzdak and V. Skokov, Phys. Lett. B 728, 662 (2014) arXiv:1309.7358 [hep-ph]].

[30] F. Gelis, E. Iancu, J. Jalilian-Marian and R. Venugopalan, Ann. Rev. Nucl. Part. Sci. 60, 463 (2010) arXiv:1002.0333 [hep-ph]].

[31] J. P. Blaizot, Rept. Prog. Phys. 80, no. 3, 032301 (2017) arXiv:1607.04448 [hep-ph]].

[32] C. Marquet, G. Soyez and B. W. Xiao, Phys. Lett. B 639, 635 (2006) hep-ph/0606233.

[33] L. McLerran and M. Praszalowicz, Annals Phys. 372, 215 (2016) arXiv:1507.05976 [hep-ph]].

[34] L. McLerran and P. Tribedy, Nucl. Phys. A 945, 216 (2016) arXiv:1508.03292 [hep-ph]].

[35] H. Mntysaari and B. Schenke, Phys. Rev. D 94, no. 3, 034042 (2016) arXiv:1607.01711 [hep-ph]].

[36] M. Praszalowicz and A. Francuz, Phys. Rev. D 92, no. 7, 074036 (2015) arXiv:1507.08186 [hep-ph]].

[37] A. Dumitru and L. D. McLerran, Nucl. Phys. A 700, 492 (2002) hep-ph/0105268.

[38] F. O. Duraes, A. V. Giannini, V. P. Goncalves and F. S. Navarra, Phys. Rev. C 94, no. 2, 024917 (2016) arXiv:1510.04737 [hep-ph]].

[39] K. Deja and K. Kutak, Phys. Rev. D 95, no. 11, 114027 (2017) arXiv:1703.07959 [hep-ph]].

[40] P. Di Francesco, M. Guilbaud, M. Luzum and J. Y. Ollitrault, Phys. Rev. C 95, no. 4, 044911 (2017) arXiv:1612.05634 [nucl-th]].

[41] R. S. Bhalerao, J. Y. Ollitrault, S. Pal and D. Teaney, Phys. Rev. Lett. 114, no. 15, 152301 (2015) arXiv:1410.7739 [nucl-th]]. 